\title{
ANÁLISIS DE LA REDISTRIBUCIÓN DE LAS REGALÍAS EN LA REGIÓN DEL LLANO. PERIODO 2011-2014
}

\author{
ANALYSIS OF ROYALtIES IN THE REgION OF THE LLANO. PERIOD 2011-2014 \\ Mauricio García Garzón* \\ Alejandra Murillo Sánchez**
}

Recibido: 11 de enero de 2017

Aceptado: 25 de abril de 2017

\section{Resumen}

Al inicio del siglo XXI, aquellas economías con producción extensiva de materias primas vieron crecer sus ingresos y sus niveles de inversión en este sector. La economía colombiana no fue la excepción, y en los últimos años, las actividades principales que han impulsado el crecimiento económico son las relacionadas con la minería y los hidrocarburos, las cuales demandan cambios en su administración. Por tal motivo, el Gobierno en el marco de la política económica y fiscal impulsó una serie de reformas que crearon el Sistema General de Regalías, con la finalidad de mejorar las condiciones de equidad social, y a su vez impulsar el desarrollo del país mediante el redireccionamiento de estas a todo el territorio nacional. El propósito de la investigación es analizar la redistribución de las regalías en la región del Llano periodo 2011-2014, a través del comportamiento del Fondo de Compensación Regional en materia de reasignación de recursos entre los departamentos productores y no productores.

Palabras clave: regalías, distribución, descentralización fiscal, equidad.

\section{Abstract}

At the beginning of the 21st century, those economies with extensive production of raw materials saw their incomes and investment levels grow in this sector. The Colombian economy was not the exception, and in recent years, the main activities that have driven economic growth are those related to mining and hydrocarbons, which demand changes in its administration. For this reason, the Government in the economic and fiscal framework policy promoted a series of reforms that created the General System of Royalties, in order to improve conditions of social and regional equity, and in turn promote the development of the country through the redirecting these to the whole national territory. The purpose of this research is to analyze of royalties in the Region of the Llano period 2011-2014, through the behavior of the Regional Compensation Fund in the reallocation of resources between the producing and non-producing departments.

Keywords: royalties, distribution, fiscal decentralization, equity.

\footnotetext{
* Economista, especialista en Gerencia Pública y Control Fiscal, magíster en Administración Pública. Docente investigador, grupo de investigación Desarrollo y Equidad de la Facultad de Economía, Fundación Universidad de América.mauricio.garcia@investigadores.uamerica.edu.co

${ }^{* *}$ Economista. Coinvestigadora, grupo de investigación Desarrollo y Equidad, Fundación Universidad de América. aleja.04.20@gmail.com
} 


\section{INTRODUCCIÓN}

"Vamos a repartir la mermelada en toda la tostada nacional",
expresión del exministro de Hacienda Juan Carlos Echeverry,
tras la aprobación final en el Congreso de la reforma constitu-
cional que crea un nuevo marco para las regalías.

RICARDO ÁVILA (2011)

Con la promulgación del Acto Legislativo 05 de 2011 y la Ley 1530 de 2012 se buscó brindar una distribución más equitativa de los recursos provenientes de las regalías, debido a que el anterior esquema permitía a las entidades territoriales productoras de recursos naturales no renovables quedarse con la mayor parte del total de las regalías y solo transferir una pequeña suma, a través de la figura del escalonamiento, al resto de los territorios del país.

Es importante decir que a nivel regional la repartición genera mayor controversia, pues las diferencias se acentúan, principalmente donde coexisten departamentos productores y no productores, como es el caso del Llano. Allí, las disparidades son muy notorias por cuanto, Arauca, Meta y Casanare que explotan los recursos naturales se encuentran mejor clasificados en los indicadores que miden las brechas de desigualdad, frente a Guaviare, Guainía, Vichada y Vaupés que no poseen dichos recursos.

Por otra parte, con la reforma se constituyó el Sistema General de Regalías (SGR), y con él, un conjunto de organismos encargados de velar por la correcta distribución de los recursos. Uno de estos organismos es el Fondo de Compensación Regional (FCR), el cual fue creado con el objetivo de disminuir las inequidades entre los diferentes territorios del país.

En ese orden de ideas, el presente documento está dirigido al análisis de la redistribución de las regalías en la región del Llano periodo 2011-2014, a partir de los avances que ha tenido el FCR en materia de reasignación de los recursos entre los departamentos productores y no productores.

El estudio se ha dividido en tres partes: en la primera se hace la revisión de algunos referentes teóricos normativos sobre la materia. En la segunda: ejercicio comparativo entre el esquema anterior de regalías y el sistema actual. Tercera: análisis de la evolución de la participación de los recursos en los departamentos de la región del Llano.

La pertinencia de la investigación radica en que Colombia se ha caracterizado por ser un país con una elevada concentración de los ingresos, tanto a nivel regional como en los estratos socioeconómicos, y la razón para crear el Fondo de Compensación Regional, según la Ley 1530 de 2012, es aminorar esas asimetrías regionales en la distribución de los recursos provenientes de regalías indirectas. Por lo tanto, adelantar investigaciones en el ámbito de la inequidad constituye una de las tareas cardinales de la academia y, particularmente, del grupo Desarrollo y Equidad de la Universidad de América.

La selección de la región del Llano para el estudio se debe a que allí se encuentran los principales yacimientos de petróleo del país. En efecto, en el corte del primer trimestre de 2015 mantiene la hegemonía en la producción de petróleo, al participar, con cerca del $67 \%$ del total nacional (Banco de la República, 2015). En consecuencia, es un territorio que recibe ingentes cantidades de regalías que son destinadas en su mayoría a los departamentos del Meta, Casanare y Arauca por ser productores; mientras que Guainía, Vichada, Guaviare y Vaupés se quedan con una parte menor del total de las regalías, acrecentando de esta forma la inequidad regional. 


\section{MÉTodo}

En este apartado se exponen los referentes teóricos, el diseño metodológico y los componentes normativos de las regalías.

\section{Referentes teóricos}

En aras de orientar la discusión con fundamentación teórica y antecedentes de trabajos similares al presente, a continuación se describen algunos referentes teóricos y conceptuales relativos a la descentralización fiscal y las economías extractivas.

\section{Descentralización fiscal}

Colombia, mediante la Constitución Política de 1991, inició formalmente un proceso de descentralización que abarca aspectos administrativos, políticos y fiscales. $\mathrm{El}$ ámbito fiscal, se refiere a la transferencia efectiva de poder político desde la administración central del Estado a los gobiernos locales, para que puedan ejercer las competencias que les fueron asignadas. Estas se han desarrollado a través de tres instrumentos: la tributación territorial, las transferencias intergubernamentales y las regalías.

Bajo esta perspectiva, el objeto de estudio se enfoca en el tercer instrumento de la descentralización fiscal, las regalías, las cuales son analizadas como un elemento clave en la consolidación de dicho proceso.

Las primeras ideas de la descentralización como forma de organización de la democracia se le atribuyen a Alexis de Tocqueville, quien desarrolló su teoría analizando la formación histórica y social de Nueva Inglaterra, cuyos principios básicos fueron adoptados después por los Estados Unidos. El autor plantea la descentralización como una forma adecuada de organización para el cumplimiento de la democracia. Según la visión de Tocqueville (citado en Finot, 2001):

La soberanía reside en el pueblo y cada individuo constituye una parte igual de esa soberanía y tiene igual derecho a participar en el gobierno. Cada individuo es igualmente capaz de autogobernarse y obedece a la sociedad no porque sea inferior a los que la dirigen, sino porque le parece útil y sabe que dicha unión no puede existir sin un poder regulador. (p. 27)

La aproximación económica a la descentralización se plantea a partir del problema de la asignación para la provisión de bienes públicos estudiado por Paul Samuelson (1954). Este autor manifiesta explícitamente que la provisión de bienes privados la determina el sistema de mercado y los bienes públicos responden a preferencias agregadas, que corresponden al suministro de bienes colectivos, refiriéndose a dos tipos de bienes:

[...] bienes ordinarios de consumo para privados, los cuales se pueden dividir en numerosos individuos [...] por otro lado, los bienes de consumo de manera colectiva, que de manera lógica el consumo de ese bien común por parte de un individuo no sustrae la posibilidad de que otro individuo igualmente lo consuma (Samuelson, 1954, p. 387).

En este sentido, Musgrave (1959), al referirse al tema de la asignación, establece que el Estado cumple tres funciones sustanciales, que corresponden a:

Estabilización macroeconómica, distribución y asignación de recursos. La primera es exclusiva del nivel nacional que cuenta con los instrumentos de política económica para desarrollarla; la segunda puede ser desarrollada por la Nación y los territorios y la tercera que comprende la provisión de bienes y servicios públicos puede ser atendida por diferentes niveles de gobierno, atendiendo a la naturaleza de dichos bienes y servicios; siendo su principal objetivo la asignación de recursos necesarios para la satisfacción de las necesidades sociales que, en común con los deseos privados, reflejan las preferencias de los individuos. (p. 118) 
Estos aspectos económicos de la descentralización se relacionan con la eficiencia de la asignación de recursos y se agrupan en teorías desarrolladas en la literatura del federalismo fiscal en los Estados Unidos, con autores como Charles Tiebout (1956) quien se refiere a la revelación de preferencias de los ciudadanos, en cuanto a la provisión de bienes públicos y la carga impositiva en el ámbito local, cabe mencionar el aporte a través de su importante artículo titulado "A Pure Theory of Local Expeditures". En dicho texto se propone un modelo mediante el cual explica que los ciudadanos revelan sus preferencias trasladándose a la comunidad que más satisfaga su demanda de bienes públicos y su respectiva carga impositiva para financiarlos. Esto es lo que se conoce como "voto con los pies". Lo anterior supone que los residentes de las jurisdicciones locales tienen mecanismos de participación política que les permite decidir sobre la provisión de bienes públicos (Wiesner, 2011).

Este enfoque, también se ha interesado por el asunto del tamaño óptimo de los gobiernos locales, puesto que no siempre la división política de los entes territoriales será la más eficiente para la provisión de bienes públicos. Con base en el conocido trabajo de Buchanan (1965), "An Economic Theory of Clubs", se ha solucionado este problema, por lo menos de manera teórica, al afirmar que el tamaño óptimo de cada nivel de gobierno se logra cuando el costo marginal de congestión es igual a la utilidad marginal por asociarse para compartir el costo del bien público

Por su parte, Wallace Oates (1972), al respecto de su "teorema de la descentralización", afirma lo siguiente:

En ausencia de economías y de efectos externos interjurisdiccionales, el nivel de bienestar será tan o más alto si los niveles de consumo óptimos (Pareto) son provistos en cada jurisdicción, en vez de serlo en cualquier nivel único y uniforme para todas las jurisdicciones, siempre que éstas coincidan exactamente con los subconjuntos de población que consumen el mismo bien público. El gobierno central no puede proporcionar un nivel de producción Pareto-eficiente para cada área. (Oates, 1972, p. 54)

Asimismo, la descentralización fiscal tiene como fin satisfacer las necesidades específicas de los diferentes sectores de la sociedad de la manera más eficiente y maximizando el bienestar económico, en ese sentido, la distribución de los recursos provenientes de las regalías se debe hacer de una manera que permita lograr la equidad entre los departamentos y municipios tanto productores como no productores.

De acuerdo a esto el economista John Rawls plantea que, el óptimo social a través de la redistribución, se puede lograr en un punto donde unos individuos ganen más que otros, si este óptimo social se obtiene en un punto superior al que estaban inicialmente, es decir, es bueno que un individuo sea más rico que otro si este último se encuentra en una mejor situación económica a la que se encontraba antes de la redistribución (Pardo, 2004).

Adicionalmente, el economista italiano Wilfredo Pareto en el siglo XIX formuló una teoría económica que buscaba hallar la explicación de las desigualdades en la distribución de la propiedad (aplicable a la inequidad en la distribución de la renta). El primer teorema de Pareto dice que el libre funcionamiento del mercado asegura la más eficiente asignación de recursos. Mientras que el segundo teorema dice que pese a ello, es necesario un cierto grado de intervención del estado, aunque sea leve, para distribuir mejor la utilidad de la sociedad (Ricciardi, 2008).

\section{Economias extractivas}

En los últimos años la explotación minera y petrolera ha impulsado el crecimiento económico en Colombia, por lo cual es importante referirse a algunos aspectos teóricos sobre el tema. Para Acosta (2009), los recursos naturales cumplen un papel muy importante en el rubro de las exportaciones de las economías Latinoamericanas, así lo señala el autor: 
La existencia de elevadas rentas hidrocarburíferas o mineras es uno de los mayores alicientes para mantener y apoyar este tipo de actividades primario-exportadoras. Los gobiernos, presionados por las urgencias cotidianas, más aún en época de crisis, buscan nuevos ingresos y, de ser posible, impulsar ambiciosos proyectos de desarrollo. A través de este esfuerzo esperan poder atender muchas de las largamente postergadas demandas sociales. (p. 135)

Lo anterior se debe en buena medida a la expansión del llamado extractivismo o neoextractivismo, que es definido por Gudynas (2013), al momento de explicar cómo se da la apropiación de los recursos naturales en América Latina:

Se pueden diferenciar distintas formas de articular el extractivismo en los estilos de desarrollo. Considerando las situaciones más recientes, ha sido apropiado identificar dos expresiones: (1) Extractivismo convencional o clásico, que sumariamente puede ser descrito como aquel que es llevado adelante sobre todo por empresas transnacionales, mientras el Estado tiene un papel secundario (usualmente brindado protección a las inversiones y las exportaciones, con bajos niveles de tributación). A su vez, se asume que los beneficios se lograrán por medio de los mecanismos de mercado, donde las exportaciones generarán efectos de derrame en el resto de la economía (como nuevos empleos), y todo ello desembocará en promover el desarrollo. (2) Neo extractivismo o nuevo extractivismo progresista, donde hay una mayor presencia del Estado de diversas maneras. En unos casos, las actividades extractivas son llevadas adelante por empresas estatales o mixtas; en otros casos, el nivel de las regalías y la imposición tributaria es más elevado; e incluso, hay mayores controles sobre la producción. Bajo esta modalidad, se entiende que los mayores beneficios no se lograrán por los simples mecanismos mercantiles, sino que es necesario contar con el concurso del Estado. (p. 8)

Continúa el autor señalando que la primera modalidad de extractivismo sigue vigente, en Colombia y México con los clásicos proyectos mineros o petroleros en manos de grandes consorcios internacionales; mientras que la segunda ha sido implementado por los gobiernos progresistas o de la nueva izquierda latinoamericana, siendo los casos más conocidos los de Venezuela, Bolivia y Ecuador que implantaron cambios en distintos sectores extractivos con profundas retóricas sociales, y como producto de esos cambios surgió el término "neo-extractivismo progresista" (Gudynas, 2013).

De otro lado, Lander (2014), al explicar el modelo de desarrollo en América Latina, hace referencia a que el extractivismo no solo forma parte de los debates internos entre los países llamados progresistas. Se trata de un modelo productivo cuyas concepciones son compartidas entre los países miembros de la Alianza Bolivariana para los Pueblos de Nuestra América-Tratado de Comercio de los Pueblos (ALBA-TCP). De esta manera el autor, trae a colación la declaración de la XII Cumbre de Jefes de Estado y de Gobierno del ALBA-TCP:

[...] manifestamos el derecho y la necesidad que tienen nuestros países de aprovechar, de manera responsable y sustentable, sus recursos naturales no renovables, los cuales cuentan con el potencial de ser utilizados como una importante fuente para financiar el desarrollo económico, la justicia social y, en definitiva, el bienestar de nuestros pueblos, teniendo claro que el principal imperativo social de nuestro tiempo -y de nuestra regiones- combatir la pobreza y la miseria. En este sentido, rechazamos la posición extremista de determinados grupos que, bajo la consigna del anti-extractivismo, se oponen sistemáticamente a la explotación de nuestros recursos naturales, exigiendo que esto se pueda hacer solamente sobre la base del consentimiento previo de las personas y comunidades que viven cerca de esa fuente de riqueza. En la práctica, esto supondría la imposibilidad de aprovechar esta alternativa y, en última instancia, comprometería los éxitos alcanzados en materia social y económica. (30 de julio de 2013, p. 10)

\section{Diseño metodológico}

Colombia, mediante la Constitución Política de 1991 inició formalmente un proceso de descentralización que abarcó aspectos administrativos, políticos y fiscales. Este último se ha desarrollado 
mediante tres instrumentos: la tributación territorial, las transferencias intergubernamentales y las regalías.

Debido a lo anterior, es importante conocer el contexto del que se pretende hablar para tener una aproximación a las teorías que se mencionan, por lo tanto, es fundamental entender lo que significa la descentralización fiscal y todo lo pertinente a esta; descentralización fiscal se refiere a la transferencia efectiva de poder político desde la administración central del Estado a los gobiernos locales, para poder cumplir con las competencias que fueron asignadas.

Bajo esta perspectiva el objeto de estudio estará enfocado al tercer instrumento de la descentralización fiscal: las regalías que se constituye en un factor fundamental en dicho proceso.

El estudio adelantado, que se resume en este artículo es de tipo exploratorio y descriptivo, apoyado analíticamente en aspectos cuantitativos; donde se realizó una amplia recolección y procesamiento de información estadística para dar evidencia real sobre los avances que ha tenido el Fondo de Compensación Regional del Llano en materia de redistribución de recursos provenientes de las regalías entre los departamentos productores y no productores.

La información estadística se tomó de fuentes oficiales entre otras: Departamento Nacional de Planeación-DNP, (Grupo de Financiamiento Territorial SGR) y del Departamento Nacional de Estadística-DANE. Los resultados cuantitativos se complementan con observaciones que obedecen a determinadas coyunturas que a juicio de los autores, explican dichos resultados.

\section{Referentes normativos}

El conjunto de normas que regulan el manejo, distribución, ejecución y control de los recursos de Regalías van desde los preceptos constitucionales, leyes, decretos y acuerdos, que al conjugarlos dan como resultado el marco normativo que reglamentan la funcionalidad del Sistema General de Regalías. A continuación se mencionan los más representativos:

Constitución Política: Artículo 332. Estipula que el Estado es el propietario del subsuelo y de los recursos naturales no renovables. Artículo 360. La explotación de un recurso natural no renovable causará, a favor del Estado, una contraprestación económica a título de regalía, sin perjuicio de cualquier otro derecho o compensación que se pacte. La ley determinará las condiciones para la explotación de los recursos naturales no renovables. Articulo 361. Los ingresos del Sistema General de Regalías se destinarán al financiamiento de proyectos para el desarrollo social, económico y ambiental de las entidades territoriales. Para efectos de cumplir con los objetivos y fines del Sistema General de Regalías, créanse los Fondos de Ciencia, Tecnología e Innovación.

Ley 141 de 1994. Crea el Fondo Nacional de Regalías, se instaura la Comisión Nacional de Regalías y señala las reglas para la liquidación, distribución y uso de las regalías.

Acto Legislativo 05 de 2011. Crea el Sistema General de Regalías (SGR)

Ley 1530 de 2012. Reglamenta el nuevo Sistema General de Regalías (SGR)

Decreto 1949 de 2012. Se reglamenta parcialmente la Ley 1530 de 2012 en materia presupuestal y se dictan otras disposiciones.

Acuerdo Comisión Rectora del Sistema General de Regalías, número 0003 de mayo de 2012, por medio del cual se adopta la regionalización para efectos del funcionamiento del SGR.

El marco normativo señalado constituye la referencia a tener en cuenta a lo largo del desarrollo de la investigación, lo que permite comprender medianamente la regulación que tiene el Sistema General de Regalías. 


\section{Resultados}

La problemática central que aborda este estudio se refiere a la distribución de los recursos de las regalías administrados por el Fondo de Compensación Regional, FCR, en la región del Llano, y su aporte a la disminución de las inequidades en dicha región a partir de la redistribución de estos recursos. Por lo anterior, en este apartado se realiza un comparativo entre el esquema anterior y el Sistema General de Regalías (SGR), y luego se analiza el proceso de la redistribución de los recursos del FCR en la región señalada. Asimismo, se plantean algunos aspectos sociales relacionados con las disparidades internas y medición de la pobreza.

\section{Comparativo entre el esquema anterior y el Sistema General de Regalías}

A continuación se hace una comparación entre el esquema anterior a la reforma del 2011 y el Sistema General de Regalías (SGR), creado mediante Acto Legislativo 05 de 2011 y la Ley 1530 de 2012. Ambos esquemas se contrastan, principalmente en los aspectos de destinación, distribución y funcionamiento (la siguiente información fue tomada de: DNP, Informes presentación del SGR, 2012).

\section{Antiguo esquema de Regalías}

Origen

- Se creó el Fondo Nacional de Regalías (FNR) mediante la Ley 141 de 1994.

- Los recursos de este fondo deberían ser destinados a la promoción de la minería, la preservación del medio ambiente y la financiación de proyectos regionales de inversión.

- Los recursos del FNR eran propiedad exclusiva de las entidades territoriales y eran recaudados y administrados por la Dirección General del Tesoro Nacional.

Distribución

- Regalías directas: $80 \%$ para municipios y departamentos donde se explotaban recursos no renovables.

- Regalías indirectas: 20 \% para el Fondo Nacional de Regalías como mecanismo nacional de distribución, al cual tenían acceso las regiones.

- Las regalías se concentraban en pocos municipios y departamentos del País.

Destinación

- Los departamentos debían destinar el $90 \%$ de sus regalías a proyectos de inversión contemplados como prioritarios en los Planes de Desarrollo municipales, siempre y cuando hubiesen cumplido los niveles de cubrimientos para las necesidades básicas de la población.

- Para poderlas invertir libremente se requería cumplir cuatro coberturas mínimas dispuestas por la Ley 141 relacionadas con mortalidad infantil (1\%), salud (100\%), educación básica (90\%), agua potable $(70 \%)$ y alcantarillado (70\%). Tras cumplir esas metas, se podían usar para otros fines.

- Esa ley rigió veinte años sin que ningún municipio lograra las cuatro coberturas. Los que cumplían dos coberturas, proporcionalmente se les permitía la inversión en unos porcentajes definidos por la ley.

Funcionamiento

- La fiscalización estuvo en manos de Planeación Nacional, que realizaba su labor a través de auditorías. Había sanciones, como congelar giros por mal uso de recursos.

- Se creó el Sistema Nacional de Gestión de Programas y Proyectos de Inversión Pública. Los órganos de control y vigilancia son la Procuraduría General de la Nación. La Contraloría General de la República, la Fiscalía General de la Nación y el DNP. El control y la vigilancia se realizaban 
a través de las Interventorías Administrativas y Financieras (IAF) que hacía la dirección de Regalías del DNP.

- La aprobación de proyectos la daba el Consejo Asesor de Regalías a aquellos viabilizados por los ministerios sectoriales.

\section{Nueva reforma a las regalías}

Origen

- Se crea el Sistema General de Regalías (SGR) mediante Acto Legislativo 05 de 2011 y Ley 1530 de 2012.

- El Sistema Nacional de Regalías reemplaza el FNR. La misma Ley 1530 establece la distribución, objetivos, fines, uso eficiente y destinación de estos ingresos.

- Los ingresos del Sistema General de Regalías se destinarán a proyectos de desarrollo social, económico y ambiental de todas las entidades territoriales.

Distribución

- Las regalías directas se distribuyen así: 2012: 50 \% de las regalías se destina a entidades territoriales (ET) productoras. 2013: $35 \%$ de las regalías se destina a ET productoras. 2014: 25\% de las regalías se destina a ET productoras. 2015 en adelante: 20\% de las regalías se destina a ET productoras.

- La regalías indirectas se distribuyen de la siguiente forma: 2012: $50 \%$ para los nuevos fondos. 2013: $65 \%$ para los nuevos fondos. 2014: $75 \%$ para los nuevos fondos. $2015: 80 \%$ para los nuevos fondos.

- Adicionalmente: hasta un 30 \% para el Fondo de Ahorro y Estabilización (FAE). 10 \% para Ahorro Pensional Territorial (FONPET). $10 \%$ para Fondo de Ciencia, Tecnología e Innovación (FCTI). 20 \% (Después de descontar FCTI, FAE y FONPET), para participación de las entidades territoriales receptoras (adicional a su participación en todos los componentes del SGR). $80 \%$ para Fondo de Desarrollo Regional y Fondo de Compensación Regional (después de descontar FCTI, FAE y FONPET).

- No solo los municipios y departamentos donde se generan las regalías serán beneficiarios, ahora todas las entidades territoriales pueden acceder a la obtención de dichos recursos siempre y cuando cumplan con lo establecido en la Ley.

- Dichos recursos serán distribuidos para todo el país.

Destinación

- Hasta un $30 \%$ de los recursos se destinarán al Fondo de Ahorro y Estabilización, con el objeto de generar ahorros para épocas de crisis, promoviendo así el carácter contracíclico de la política económica y mantener estable el gasto público a través del tiempo.

- E1 $10 \%$ de las regalías podrán destinarse a proyectos de ciencia, tecnología e innovación.

- Inversión hasta un 40 \% para proyectos de impacto local a través del Fondo de Compensación Regional (FCR).

- Las regalías serán destinadas para ahorro e inversión, así: generación de ahorro público, generación de ahorro para pasivo pensional; financiación de proyectos para el desarrollo social, económico y ambiental de las entidades territoriales; inversión en educación, ciencia, tecnología e innovación; fiscalización de la exploración y explotación de yacimientos; y conocimiento y cartografía geológica del suelo.

Funcionamiento

- Se asigna un $2 \%$ del total de las regalías para la fiscalización, que estará en cabeza del Ministerio de Minas, el cual establecerá un esquema integral de vigilancia desde la producción. 
- Respecto a la vigilancia y el control, se crea el Sistema de Monitoreo, Seguimiento, Control y Evaluación (SMSCE) administrado por el DNP. Los órganos de control y vigilancia son la Procuraduría General de la Nación, la Contraloría General de la República, la Fiscalía General de la Nación y el DNP.

- La interventoría que se ejerza será administrativa, técnica, financiera, contable y jurídica, contratada según el Estatuto Anticorrupción.

- Respecto a la aprobación de proyectos: las regalías son de libre inversión, pero esa inversión se define en los Órganos Colegiados de Administración y Decisión (OCAD), los cuales designarán el ejecutor de proyectos.

- Todos los recursos del SGR financiaran proyectos de inversión aprobados por los OCAD. E1 SGR podrá financiar estudios y diseños. El SGR tiene su propio banco de proyectos de impacto local a través del Fondo de Compensación Regional (FCR).

- El SGR maneja su propio presupuesto, el cual es aprobado por dos años. Los recursos del sistema se incorporan por decreto en los presupuestos de las entidades beneficiarias de las regalías directas.

E1 comparativo desarrollado permite apreciar las diferencias sustantivas entre el SGR y el antiguo esquema, especialmente en materia de distribución y destinación de recursos, lo que permite obtener elementos de discusión y análisis para el presente estudio.

En materia de instituciones, el principal cambio que se da con la reforma es la liquidación del Fondo Nacional de Regalías y la constitución del Sistema General de Regalías como órgano máximo de administración de los recursos, el cual, a su vez, incluye varios fondos y organismos que constribuyen a realizar la distribución de los recursos hacia las entidades territoriales.

Con la reforma se cambió la distribución de $80 \%$ de regalías directas para entidades territoriales productoras y $20 \%$ para las entidades territoriales no productoras. En el 2015 la distribución se revirtió, pues a partir de este año es de $80 \%$ de regalías indirectas y $20 \%$ de regalías directas, como medida para mitigar la concentración de los recursos.

Es preciso mencionar que el principal avance de la reforma está en la parte de la distribución, pues se crean fondos en diversos sectores que constituyen organismos enfocados hacia la redistribución, la equidad y la competitividad. En la actualidad son muchos los municipios que han presentado y aprobado proyectos; adicionalmente, algunos de los proyectos que ha financiado el SGR son de gran envergadura para las regiones, pues constituyen grandes avances, tales como, la electrificación rural y la construcción de hospitales, colegios etc.

\section{Redistribución de los recursos girados por el Fondo de Compensación Regional en la región del Llano}

De acuerdo con el artículo 34 de la Ley 1530 de 2012, el Fondo de Compensación Regional (FCR) tiene como objeto financiar los proyectos de desarrollo de impacto regional o local en las entidades territoriales más pobres del país, acordados entre el Gobierno Nacional y las entidades territoriales, según los criterios señalados en el inciso noveno del artículo 361 de la Constitución Política de 1991.

La razón de ser del FCR es corregir las asimetrías regionales que presenta Colombia, especialmente en aquellos departamentos y municipios ubicados en la periferia del país, a la que pertenece la región Llanera, por tanto, luego de la reforma a las regalías se espera que con estos recursos sean ejecutados proyectos de inversión que mejoren la calidad de vida de sus habitantes.

De igual manera, se espera que con el FCR aquellas regiones del país con mayores niveles de inequidad obtengan recursos importantes para ser destinados a la reducción de la misma. En la 
figura 1 se aprecian las regiones que más se han visto favorecidas con este fondo, ya que ostentan altos índices de pobreza y desigualdad. Tal es el caso de la región Caribe y Pacífico.

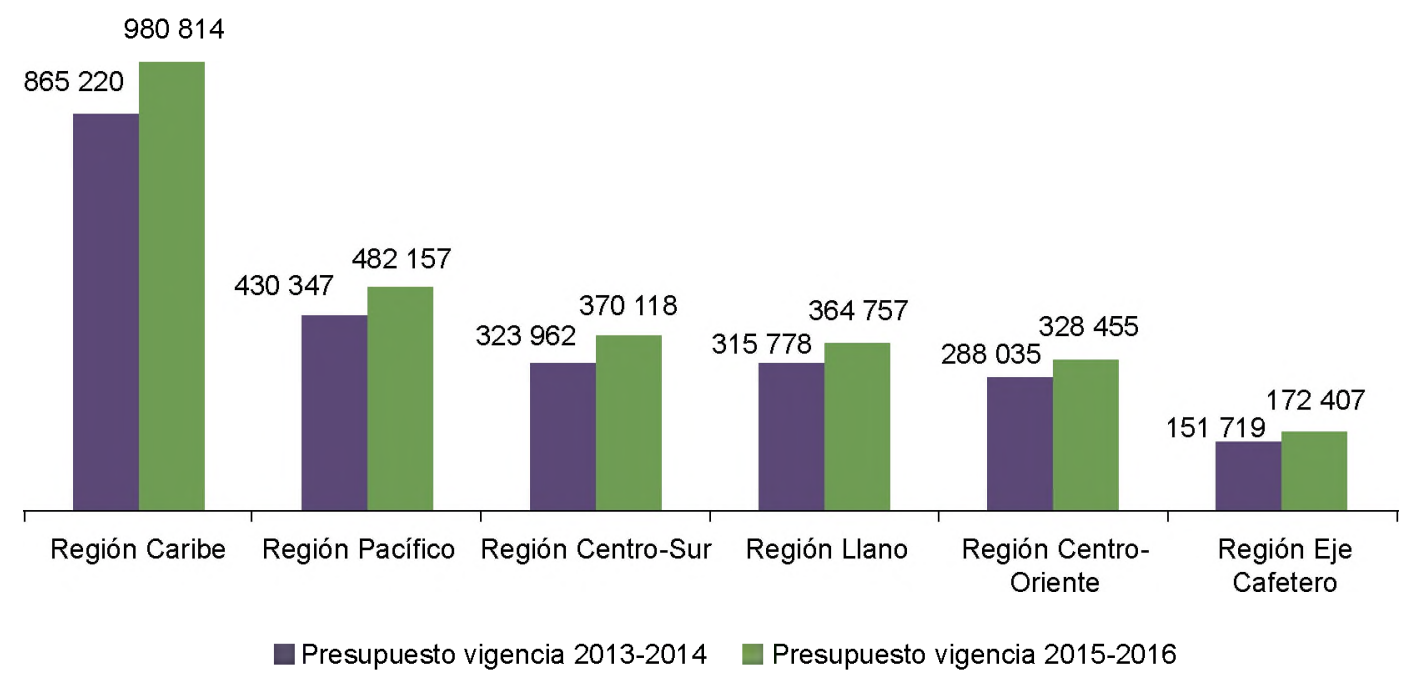

Figura 1. Presupuesto del FCR por región (millones de pesos), 2013-2016.

Fuente: elaboración propia, con datos del Grupo de Financiamiento Territorial SGR-DNP

Como se observa en la figura, la región Caribe se lleva una porción considerable de los recursos, seguida por la región del Pacífico. Es consabido que en esas dos regiones se encuentra el grueso de la población más pobre del país, en consecuencia se justifica que se dirija hacia allá ellos una cuantía superior por concepto de regalías indirectas vía FCR. La región del Llano se encuentra en un punto intermedio, teniendo también numerosas necesidades sociales por suplit.

Una vez referido el contexto nacional y señaladas las finalidades del FCR, se analiza cómo dicho Fondo ha venido cumpliendo su papel al redistribuir los recursos provenientes de las regalías entre los departamentos de la región Llanera.

En este sentido es realizado el siguiente ejercicio: se toman los ingresos departamentales por regalías, tanto por vía directa como indirecta giradas por el FCR, con el fin de observar la evolución que ha tenido la participación de los departamentos productores y no productores en estos recursos. En la tabla 1 se muestra los resultados.

Como se puede observar en la tabla 1, para el año 2012 los departamentos productores, Arauca, Casanare y Meta vieron aumentar sus recursos en 167408 millones de pesos, soportado por el incremento en los ingresos del departamento del Casanare. Para el año 2013, estos mismos departamentos dejaron de recibir 593721 millones de pesos y para la vigencia del 2014, la disminución fue de 438074 millones de pesos.

E1 panorama para los departamentos no productores, Guainía, Guaviare, Vaupés, y Vichada, que en el esquema anterior no recibían recursos por concepto de regalías, se torna en algo diferente, por cuanto para el año 2012 recibieron la suma de 67189 millones de pesos. Para el año 2013, estos mismos departamentos obtuvieron 67018 millones de pesos y finalmente para el año 2014 el valor girado aumentó a 76296 millones de pesos.

De otro lado, los departamentos de Meta, Casanare y Arauca siguen concentrando la mayor parte de los ingresos de las regalías, por cuanto a 2014 éstos representaron el 51.9\%, $29.5 \%$ y $9.9 \%$ de los recursos de la región respectivamente, sumando el $91.4 \%$ del total; frente a los departamentos 
no productores que para ese mismo año recibieron: Guainía $2.0 \%$, Guaviare $2.3 \%$, Vaupés $1.9 \%$ y Vichada $2.5 \%$ lo que representa el $8.6 \%$ de los ingresos recibidos por la región.

Tabla 1. Ingresos por departamentos antes y después de la reforma (millones de pesos) y participación porcentual en la región del Llano

\begin{tabular}{|c|c|c|c|c|c|c|c|c|}
\hline \multirow[b]{2}{*}{ Departamento } & \multicolumn{2}{|c|}{2011} & \multicolumn{2}{|c|}{2012} & \multicolumn{2}{|c|}{2013} & \multicolumn{2}{|c|}{2014} \\
\hline & 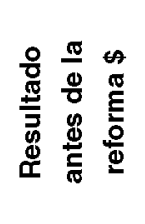 & 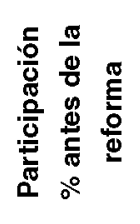 & 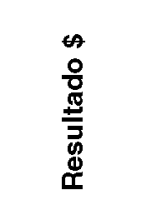 & 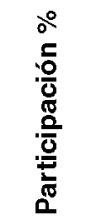 & 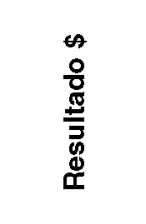 & 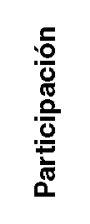 & 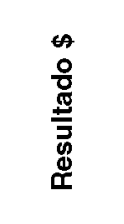 & 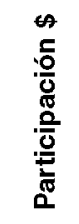 \\
\hline \multicolumn{9}{|l|}{ Productores } \\
\hline Arauca & 294072 & 17.6 & 215862 & 11.3 & 145014 & 1.1 & 87271 & 9.9 \\
\hline Casanare & 322154 & 19.3 & 579666 & 30.4 & 377865 & 28.8 & 260427 & 29.5 \\
\hline Meta & 1054007 & 63.1 & 1042113 & 54.7 & 721041 & 55.0 & 458148 & 51.9 \\
\hline $\begin{array}{l}\text { Total } \\
\text { productores }\end{array}$ & 1670233 & 100.0 & 1837641 & 96.5 & 1243920 & 94.9 & 805846 & 91.4 \\
\hline \multicolumn{9}{|l|}{ No productores } \\
\hline Guainia & 0 & 0 & 15068 & 0.8 & 15018 & 1.1 & 17352 & 2.0 \\
\hline Guaviare & 0 & 0 & 17975 & 0.9 & 17976 & 1.4 & 20348 & 2.3 \\
\hline Vaupés & 0 & 0 & 14577 & 8.0 & 14577 & 1.1 & 16442 & 1.9 \\
\hline Vichada & 0 & 0 & 19569 & 1.0 & 19447 & 1.5 & 22154 & 2.5 \\
\hline $\begin{array}{l}\text { Total no } \\
\text { productores }\end{array}$ & 0 & 0 & 67189 & 3.5 & 67018 & 5.1 & 76296 & 8.7 \\
\hline Total girado & 1670233 & 100.0 & 1904830 & 100.0 & 1310938 & 100.0 & 882142 & 100.0 \\
\hline
\end{tabular}

Fuente: elaboración propia, con datos del Grupo de Financiamiento Territorial SGR-DNP

El grueso de los recursos recibidos por los departamentos no productores llega por vía de regalías indirectas giradas por el Fondo de Compensación Regional, equivalente al $95 \%$ del total, y solamente el $5 \%$ corresponde a regalías directas giradas por el SGR con destino a las comunidades negras, afrocolombianas, raizales, palenqueras, comunidades indígenas y resguardos indígenas, en cumplimiento del artículo 40 de la Ley 1530 de 2012.

Asimismo, se pueden observar en la tabla 2 los cambios porcentuales en la participación de los departamentos productores (lo que dejan de recibir), versus los cambios porcentuales en la participación de los departamentos no productores (lo que reciben) en el período 2012-2014.

A partir de la reforma que crea el Sistema General de Regalías (SGR) y el Fondo de Compensación Regional (FCR), los departamentos no productores ganaron $8.7 \%$ participación en la distribución de las regalías en la región del Llano, cifra que dejan de percibir los departamentos productores. El departamento más beneficiado fue Vichada, que ganó $2.5 \%$, y el más afectado fue el Meta, que perdió $11.2 \%$. El año 2014 fue el más significativo en el aumento de recursos para los departamentos no productores, con el $3.5 \%$.

La cifra del 8.7 representa el indicador de redistribución de los recursos de las regalías entre los departamentos de la región del Llano durante el período analizado, el cual mide en qué proporción se están corrigiendo las asimetrías presentadas en la distribución de dichos recursos; no obstante, teniendo en cuenta que el acceso a las regalías indirectas depende de la capacidad de presentar proyectos viables, se siguen concentrando los recursos en los departamentos productores, lo que hace 
que la "mermelada" de las regalías no se esparza uniformemente en toda la tostada del territorio nacional, generando grumos en buena parte de ésta.

Tabla 2. Ingresos por departamentos. Cambios porcentuales en la participación en la región del Llano después de la reforma, 2012-2014

\begin{tabular}{lcccc}
\hline & \multicolumn{3}{c}{ Cambios porcentuales } & Total \\
\cline { 2 - 4 } & 2012 & 2013 & 2014 & periodo \\
\hline Productores & & & -1.17 & -7.71 \\
\hline Arauca & -6.27 & -0.27 & 0.7 & 10.23 \\
Casanare & 11.14 & -1.61 & -11.18 \\
Meta & -8.4 & 0.29 & -3.07 & -8.66 \\
\hline Total productores & -3.53 & -1.59 & -3.54 & 1.96 \\
\hline No productores & & & 2.31 \\
\hline Guainía & 0.79 & 0.35 & 0.82 & 1.87 \\
Guaviare & 0.94 & 0.43 & 0.94 & 2.52 \\
Vaupés & 0.77 & 0.35 & 0.75 & 8.66 \\
\hline Vichada & 1.03 & 0.46 & 1.03 & 3.54 \\
\hline Total no productores & 3.53 & 1.59 & & 2 \\
\hline
\end{tabular}

Fuente: elaboración propia, con datos del Grupo de Financiamiento Territorial SGR-DNP

\section{Aspectos sociales de la región del Llano}

Es importante mencionar, que a través del Fondo de Compensación Regional se pretende contribuir a cerrar las brechas de desigualdad, debido a que los recursos no se han distribuido homogéneamente en todo el territorio Nacional. Particularmente en la región del Llano, estas disparidades son más notorias: Guaviare está clasificado en la brecha de desigualdad alta y los departamentos de Guainía, Vichada y Vaupés se encuentran en medio alta. Los mejores son Arauca y Meta que están en las brechas medio baja y baja respectivamente (DNP, 2014)

De otro lado, en la región de los Llanos se presenta para el año 2013 un índice de pobreza multidimensional (IPM) de $64 \%$; cifra que se encuentra por encima del promedio nacional que es $48.97 \%$. Aspecto de por si negativo, al cual se le suma que todos los departamentos de la región se ubican por encima de este promedio. Los departamentos con mayores incidencias en pobreza son Vichada (84.3\%), Vaupés (77.8\%), Guainía (78.8\%) y Guaviare (75\%). Los departamentos con menores incidencias son Meta (51.1\%) Arauca (59.2\%) y Casanare (56.75\%) (DNP, 2014).

Para cerrar las brechas es necesario que los departamentos de la Región ejecuten irrestrictamente las metas contenidas en sus planes de desarrollo, con el propósito de generar avances intrarregionales y poder disminuir la desigualdad social.

En este marco descrito, los recursos del SGR asignados a los diferentes fondos deberán financiar proyectos de inversión que cierre las brechas, reduzcan el índice de pobreza y mejoren la competitividad de las diferentes regiones del país. 


\section{Desempeño del Fondo de Compensación Regional del Llano}

Los proyectos de inversión deben ser presentados por los municipios y departamentos a los OCAD respectivos para su aprobación. Para darle viabilidad a los proyectos, se podrán apoyar en personas jurídicas públicas y privadas, o personas naturales con experiencia y reconocida trayectoria e idoneidad, también son los encargados de evaluar, priorizar la viabilidad de los proyectos, designar ejecutores para los proyectos, conformar comités consultivos, entre otros. El estado de los proyectos presentados y su trámite se muestran a continuación con corte al mes de agosto de 2015 .

Tabla 3. Proyectos presentados por región y su estado de trámite (2012 - agosto 2015)

\begin{tabular}{|c|c|c|c|c|c|c|c|c|c|c|}
\hline $\begin{array}{r}\text { Estado del } \\
\text { proyecto }\end{array}$ & $\begin{array}{l}\frac{0}{0} \\
\frac{0}{0} \\
\text { 은 } \\
\text { 흔 }\end{array}$ & 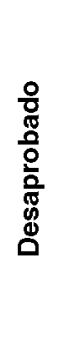 & 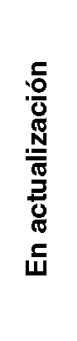 & 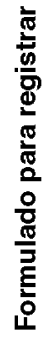 & 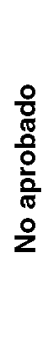 & $\begin{array}{l}\frac{0}{0} \\
\frac{\pi}{2} \\
\frac{0}{2} \\
\frac{0}{2}\end{array}$ & 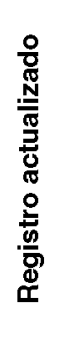 & 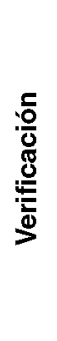 & 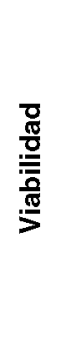 & $\begin{array}{l}\text { एक } \\
\text { 。 }\end{array}$ \\
\hline Región Caribe & 2.148 & 50 & 371 & 27 & 25 & 10 & 20 & 25 & 101 & 3.023 \\
\hline Región Centro- Oriente & 1.467 & 18 & 54 & 27 & 20 & 11 & 27 & 26 & 90 & 1.740 \\
\hline $\begin{array}{l}\text { Región Centro- sur- } \\
\text { Amazonas }\end{array}$ & 1.143 & 50 & 439 & 28 & 9 & 21 & 7 & 28 & 48 & 1.773 \\
\hline Región eje cafetero & 1.010 & 47 & 174 & 23 & 2 & 5 & 8 & 17 & 40 & 1.326 \\
\hline Región Pacífico & 1.374 & 57 & 396 & 31 & 18 & 4 & 10 & 14 & 83 & 1.987 \\
\hline Región Llanos & 1.202 & 64 & 216 & 6 & 3 & 1 & 3 & 4 & 37 & 1.536 \\
\hline
\end{tabular}

Fuente: SGR (GESPROY) 2015.

Como se puede apreciar en la tabla 3, la región del Llano se ubica como penúltima en cuanto al número de proyectos presentados con un $13.5 \%$ del total y antepenúltima en los aprobados, con un $14.4 \%$ del total. Lo anterior se presenta porque no todos los municipios y departamentos poseen la capacidad técnica con relación a su capital humano. En este sentido, los criterios que se usan para aprobar los proyectos son rigurosos y deben ceñirse al cumplimiento de elementos básicos de evaluación, como son: la forma en que se enmarca en los planes de desarrollo local, departamental y nacional; las fuentes de financiación; la buena estructuración del proyecto, entre otros. De esta manera el Sistema hace uso de variables medibles y verificables que se entregan a los miembros de los OCAD, para que con criterios objetivos soporten sus decisiones. En caso de que los proyectos no cumplan con alguno de los elementos mencionados no se aprueban. Por lo tanto, es imprescindible contar con personal cualificado en el tema, que posibilite la elaboración de proyectos que cumplan con los requisitos que establece el DNP y los OCAD respectivos. Otra alternativa para encauzar los proyectos hacia su aprobación es que el Gobierno Nacional haga un acompañamiento permanente a las regiones mediante el envío de técnicos y expertos en el tema de formulación y evaluación de proyectos, priorizando en aquellos municipios y regiones de menor categorización. 
Tabla 4. Número de proyectos por departamento (2012 - agosto de 2015)

\begin{tabular}{|c|c|c|c|c|c|c|c|c|c|c|}
\hline $\begin{array}{r}\text { Estado del } \\
\text { proyecto }\end{array}$ & 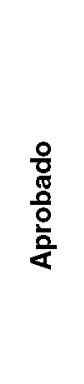 & 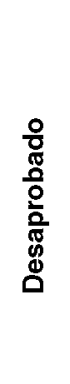 & 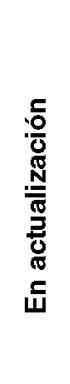 & 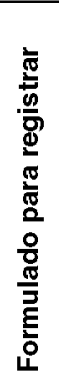 & 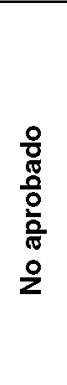 & $\begin{array}{l}\frac{0}{0} \\
\frac{\pi}{2} \\
\frac{0}{2}\end{array}$ & 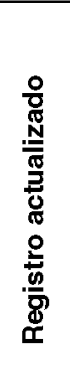 & 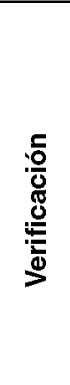 & $\begin{array}{l}\frac{7}{0} \\
\frac{\pi}{0} \\
\frac{\overline{0}}{\overline{0}} \\
\stackrel{0}{\frac{\pi}{5}}\end{array}$ & 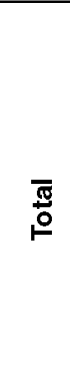 \\
\hline Arauca & 281 & 7 & 14 & 3 & 1 & 0 & 0 & 0 & 1 & 307 \\
\hline Casanare & 334 & 18 & 54 & 2 & 1 & 0 & 0 & 2 & 10 & 421 \\
\hline Guainía & 21 & 3 & 9 & 0 & 0 & 0 & 0 & 0 & 2 & 35 \\
\hline Guaviare & 41 & 2 & 9 & 0 & 0 & 0 & 0 & 0 & 2 & 54 \\
\hline Meta & 469 & 31 & 99 & 0 & 1 & 0 & 3 & 2 & 10 & 615 \\
\hline Vaupés & 23 & 3 & 21 & 1 & 0 & 0 & 0 & 0 & 3 & 51 \\
\hline Vichada & 33 & 0 & 10 & 0 & 0 & 1 & 0 & 0 & 9 & 53 \\
\hline
\end{tabular}

Fuente: SGR (GESPROY) 2015.

La tabla 4 muestra las disparidades que se dan en la presentación y trámite de proyectos por parte de los departamentos que conforman la Región llanera. Mientras el Meta ha presentado desde la reforma del régimen de regalías un total de 615 proyectos, el Guainía solamente lo ha hecho con 35 proyectos para el mismo periodo. También, el Meta aparece como el departamento que tiene más proyectos desaprobados. Teniendo en cuenta esas estadísticas resulta claro que la realidad en la asignación de recursos provenientes de regalías no ha cambiado mucho desde su reforma, pues antes, los departamentos del Meta, Casanare y Arauca eran los principales receptores de estos recursos de manera directa, hoy lo siguen siendo en forma directa e indirecta.

\section{Comentarios}

Con la nueva distribución de los recursos de las regalías, los departamentos productores han disminuido sus ingresos por regalías directas, en favor de los departamentos no productores; no obstante, teniendo en cuenta que para acceder a las regalías indirectas se deben presentar proyectos que sean viables, estos departamentos no tienen la capacidad institucional ni el personal cualificado para elaborarlos y en consecuencia no son aceptados en los OCAD correspondientes, por lo cual se seguirá presentando la mencionada concentración de dichos recursos.

Los departamentos de Meta, Casanare y Arauca siguen concentrando la mayor parte de los ingresos de las regalías, sumando para el año 2014 el $91.4 \%$ del total; frente a los departamentos no productores que para ese mismo año recibieron $8.6 \%$ de los recursos recibidos por la región.

A partir de la creación del Fondo de Compensación Regional (FCR), los departamentos no productores ganaron participación en la distribución de las regalías en la región del Llano. Vichada se constituyó en el departamento con mayor aumento (2.5\%). El año más representativo en este incremento fue el 2014 con el $3.5 \%$. 
E1 indicador de redistribución de los recursos de las regalías entre los departamentos de la región del Llano para el período 2012-2014 se situó en $8.7 \%$, lo que determina en qué proporción se están corrigiendo las asimetrías presentadas en la distribución de estos recursos. De acuerdo con la finalidad del FCR, esta redistribución comienza a impulsar la equidad social de la región, aunque de manera incipiente.

El aporte del FCR para disminuir las inequidades podría ser mayor en la medida que, por una parte, se brinde desde el Gobierno capacitaciones especializadas a los municipios y departamentos para que logren presentar proyectos viables, y por otra, se le asigne un porcentaje mayor de recursos de regalías indirectas al Fondo de Compensación Regional, teniendo en cuenta su papel redistribuidor.

Se destaca el papel desempeñado por el FCR en la región llanera, al materializar proyectos referentes a la inclusión social, superación de la pobreza y disminución de la desigualdad; así como, en la viabilización de proyectos dirigidos a las comunidades indígenas que habitan en la región, lo cual constituye un esfuerzo para aminorar la inequidad y transitar hacia la inclusión social. Además, el FCR ha ejecutado los presupuestos entregados desde los inicios del mismo en 2012 casi en su totalidad, es decir, se ha dado cumplimiento a la ejecución presupuestal de las partidas destinadas específicamente al FCR.

Desde la perspectiva del marco teórico planteado en la presente investigación sobre el federalismo fiscal y la descentralización fiscal, se puede afirmar que los principios básicos de estos no se están cumpliendo en materia de toma de decisiones, en tanto que, la determinación sobre la aprobación o desaprobación de los proyectos presentados por las entidades territoriales le corresponde a los OCAD, los cuales son conformados por ministros (como representantes del gobierno nacional), la mayoría de gobernadores y los alcaldes (como representantes de los intereses locales) que tienen poca participación. Esta situación afecta el proceso de descentralización fiscal, pues es una muestra de recentralización de las decisiones.

Por lo anterior, se están contraviniendo los conceptos desarrollados por varios autores: Tiebout (1956) quien establece la premisa de que lo local, al estar más cercano al individuo, puede captar con mayor precisión las necesidades de la comunidad; Musgrave (1959), quien establece que la función de asignación resulta ser objeto de la descentralización, dado que las administraciones locales y regionales poseen mejor información para optimizar el gasto y de esta manera responder a las necesidades de su población. Oates (1972) determina que un país está profundizando su proceso descentralización cuando sus entidades territoriales gozan de un cúmulo de competencias cada vez mayor, y requieren a su vez una mayor transferencia de recursos para llevarlas a cabo; Pareto menciona que la descentralización puede determinar un nivel de bienestar más alto, a través de la provisión en cada jurisdicción de los niveles de consumo, por lo que el gobierno central no puede proporcionar un nivel de producción "Pareto-eficiente" para cada región.

Mientras que el acceso a las regalías indirectas dependan de la capacidad de presentar proyectos viables y no se fortalezcan las capacidades institucionales de los entes territoriales, se seguirá presentando alta concentración de los recursos de las regalías en los departamentos productores; lo cual hace que la "mermelada" de las regalías no se extienda a lo largo y ancho de la "tostada" del territorio nacional, creando grumos en buena porción de ella, y de esta manera se compromete el cumplimiento de la finalidad del FCR, que es promover la equidad social y regional, para que las regiones del país alcancen los promedios nacionales en materia de calidad de vida. 


\section{ReCOMENDACIONES}

Es necesario que la academia continúe desarrollando el tema de las regalías con el fin de contribuir al debate intelectual y político. En estudios posteriores se podrían estudiar otras regiones del país, o la misma región del Llano, pues en el transcurso de unos años habrá más información con la cual se obtendrán más conclusiones sobre el desempeño del Fondo de Compensación Regional.

En aras de impulsar la formulación de proyectos en las regiones y en el país en general, es importante fortalecer la cualificación del capital humano responsable de preparar y presentar dichos proyectos a los OCAD respectivos. De esta forma, al cumplir con los estándares de calidad requeridos, se minimizan las posibilidades de que sean rechazados o declarados no viables.

El propósito para el cual se creó el Sistema General de Regalías puede verse comprometido, por el comportamiento de los precios internacionales de los commodities, por lo que se recomienda realizar, por parte de las entidades gubernamentales y la academia misma, un constante monitoreo al sistema en su conjunto, y si es el caso, proponer las reformas correspondientes.

\section{REFERENCIAS}

Acosta, A. (2009). La maldición de la abundancia. (1a ed.). Quito: Ediciones Abya-Yala.

Ávila, R. (12 de junio de 2012) Editorial. Portafolio. Recuperado de http://www.portafolio.co/ opinion/editorial/repartir-mermelada-133892.

Banco de la República. (2015). Boletín Económico Regional. I trimestre de 2015. Suroriente. Recuperado de http://www.banrep.gov.co/sites/default/files/publicaciones/archivos/ber_suroriente_tri1_2015.pdf

Buchanan, J. (1965). An Economic Theory of Clubs. Economica, 32(125), 1-14.

Departamento Nacional de Planeación. (2007). Actualización de la Cartilla "Las Regalías en Colombia". Bogotá: DPN.

Departamento Nacional de Planeación (2014). Diálogo regional para la construcción del plan nacional de desarrollo 2014-2018. Recuperado de https://colaboracion.dnp.gov.co/CDT/Prensa/ Presentaciones/Encuentro\%20Regional\%20In\%C3\%ADrida\%20211114.pdf

Finot, I. (2001, mayo). Descentralización en América Latina: Teoria y práctica. Santiago de Chile: ILPES

Gudynas, E. (2013). Extracciones, extractivismos y extrahecciones. Un marco conceptual sobre la apropiación de los recursos naturales. Recuperado de http://ambiental.net/wp-content/ uploads/2015/12/GudynasApropiacionExtractivismoExtraheccionesOdeD2013.pdf

Lander, E. (2014). El Neoextractivismo como modelo de desarrollo en América Latina y sus contradicciones. Recuperado de https://mx.boell.org/sites/default/files/edgardolander.pdf

Musgrave, (1959). The theory of public finance: a study in public economy. New York: Graw-Hill.

Oates, W. (1972). Federalismo fiscal. Madrid: Instituto de Estudios de Administración Local.

Pardo, J., y Rivera, M. (2004). Economía y Mercado I. Producción y distribución. Escuela Superior de Administración Pública. Programa de Administración. Pública Territorial.

Samuelson, P. (1954). The Pure Theory of Public Expenditure. The Review of Economics and Statistics, 36(4), 387-389.

Wiesner, E. (2011). Descentralización y federalismo fiscal. Bogotá: Departamento Nacional de Planeación. 


\section{NORMAS CITADAS}

Constitución Política de Colombia (1991). Título XII. Del Régimen Económico y de la Hacienda Pública. Recuperado de http://www.corteconstitucional.gov.co/inicio/Constitucion\%20 politica\%20de\%20Colombia\%20-\%202015.pdf

Ley 715 de 2001. Recuperado de http://www.mineducacion.gov.co/1621/articles-86098_archivo_pdf.pdf

Ley 1530 de 2012. Recuperado de http://www.alcaldiabogota.gov.co/sisjur/normas/Norma1. jsp?i $=47474$

Acuerdo 0003 del 22 de mayo de 2012. Recuperado de https:/www.sgr.gov.co/Normativa/ AcuerdosComisi\%C3\%B3nRectora/AcuerdosComisi\%C3\%B3nRectora2012.aspx

Acuerdo 0013 del 19 de octubre de 2012. Recuperado de https://www.sgr.gov.co/Normativa/ AcuerdosComisi\%C3\%B3nRectora/AcuerdosComisi\%C3\%B3nRectora2012.aspx 
